\title{
External validation of nomogram to predict inguinal lymph node metastasis in patients with penile cancer and clinically negative lymph nodes
}

Carlos Vaz de Melo Maciel ${ }^{1}$, Roberto Dias Machado ${ }^{1}$, Mariana Andozia Morini ${ }^{1}$, Pablo Aloisio Lima Mattos ${ }^{2}$ Ricardo dos Reis ${ }^{1}$, Rodolfo Borges dos Reis ${ }^{1}$, Gustavo Cardoso Guimarães ${ }^{3}$, Isabela Werneck da Cunha ${ }^{3}$, Eliney Ferreira Faria ${ }^{1}$

${ }^{1}$ Departamento de Urologia, Hospital do Câncer de Barretos, Barretos, SP, Brasil; ${ }^{2}$ Associação Piauiense de Combate ao Câncer, Teresina, PI. Brasil; ${ }^{3}$ Fundação Antonio Prudente, A. C. Camargo Cancer Center, São Paulo, SP, Brasil

\section{ABSTRACT}

Introduction: Penile cancer (PC) occurs less frequently in Europe and in the United States than in South America and parts of Africa. Lymph node (LN) involvement is the most important prognostic factor, and inguinal LN (ILN) dissection can be curative; however, ILN dissection has high morbidity. A nomogram was previously developed based on clinicopathological features of PC to predict ILN metastases. Our objective was to conduct an external validation of the previously developed nomogram based on our population.

Materials and methods: We included men with cNO ILNs who underwent ILN dissection for penile carcinoma between 2000 and 2014. We performed external validation of the nomogram considering three different external validation methods: $k$-fold, leave-oneout, and bootstrap. We also analyzed prognostic variables. Performance was quantified in terms of calibration and discrimination (receiver operator characteristic curve). A logistic regression model for positive ILNs was developed based on clinicopathological features of PC.

Results: We analyzed 65 men who underwent ILN dissection (cN0). The mean age was 56.8 years. Of 65 men, 24 (36.9\%) presented with positive LNs. A median 21 ILNs were removed. Considering the three different methods used, we concluded that the previously developed nomogram was not suitable for our sample.

Conclusions: In our study, the previously developed nomogram that was applied to our population had low accuracy and low precision for correctly identifying patients with PC who have positive ILNs.

\section{ARTICLE INFO}

Eliney Ferreira Faria

https://orcid.org/0000-0002-8297-3417

\section{Keywords:}

Nomograms; Lymph; Lymphatic

Metastasis; Penile Neoplasms

Int Braz J Urol. 2019; 45: 671-8

Submitted for publication:

November 08, 2018

Accepted after revision:

February 24, 2019

Published as Ahead of Print:

May 02, 2019

\section{INTRODUCTION}

Penile cancer is less frequent in Europe and in the United States than in other regions of the world. For instance, in South America and parts of
Africa, the incidence of PC is high, where it can accounts for $1-2 \%$ of malignant diseases $(1,2)$ in men and represents an important public health issue.

Nodal involvement is the most important prognostic factor (3) in penile cancer, and curren- 
tly available noninvasive staging methods have low sensitivity for detection of regional lymph node (LN) involvement. Optimal management of patients who are clinically node-negative (cNo) is still debated (4).

Inguinal LN dissection (ILND) can be curative; however, the procedure has high morbidity rates with respect to short- and long-term complications (5). On the other hand, surveillance strategies in patients with cNO disease (intermediate/ high risk, T1b or greater) have been associated with worse survival rates in recent non-randomized, retrospective studies (6-8).

Other alternatives, such as ultrasound-guided fine-needle biopsy, dynamic sentinel node biopsy (DSNB) $(9,10)$ or minimally invasive approaches, including pure laparoscopic or robotic-assisted ILND (11-14), have been described. However, these methods are dependent on technology, expertise, and have high costs; moreover, their advantages remain unclear.

Nomograms are low cost prediction tools for quantifying individual risk based on prognostic factors, which could be helpful in developing countries. For several cancers, nomograms might provide more precise prediction compared with the traditional tumor-node-metastasis (TNM) classification. Zhu et al. (15) developed a nomogram based on clinicopathological features (T stage, grade, lymphovascular invasion, p53 expression) of penile cancer and clinically negative inguinal LNs (ILNs). This nomogram was designed to predict ILN metastases in squamous cell carcinoma of the penis, to spare patients from unnecessary ILND, especially those living in poor countries. However, the nomogram still requires external validation. The objective of this study was to conduct external validation of the nomogram developed by $\mathrm{Zhu}$ et al. (15), based on our population.

\section{MATERIALS AND METHODS}

After receiving Institutional Review Board and ethics committee approval for the study, we included 65 men between 2000 to 2014 who underwent ILND as a part of treatment for primary penile squamous cell carcinoma and who presented with cNo stage disease preoperatively. The definition of cNo in our study was nonpalpable ILN. All patients were classified according to the European Association of Urology Risk Classification (EAURC) of penile cancer (16). In our routine practice, we normally suggest bilateral ILND for all patients who are classified as intermediate or high risk, according to the EAURC (17) ILND is generally performed 2-6 weeks after primary disease resection. The time from presentation to primary disease treatment was unavailable because this information was unreliable in the medical records. All pathological reviews were performed by an uropathologist using primary tumor slides. Tumor stage was assigned using the 2002 American Joint Committee on Cancer (TNM) system (18). T2 stage was divided into two subgroups, as in the nomogram by Zhu et al., (15) based on depth of invasion (T2a and T2b, corpus spongiosum and corpus cavernosa involvement, respectively). We used T1a and T1b jointly as category T1 and used Broders system to classify the histologic grade (18) in the same manner as in the nomogram. Lymphovascular invasion and p53 expression (cut-off expression of 20\%) (19) were also evaluated in our study. We collected data from patients at three different instituitions, then we performed external validation of the nomogram by Zhu et al. (15).

\section{Statistical analysis}

Data were analyzed using frequency and percentages for qualitative variables and medians and ranges for continuous variables. Comparisons between groups were performed using the chi-square or Fisher's exact test for qualitative variables and the Mann-Whitney test for quantitative variables. Performance was further quantified in terms of calibration and discrimination. Discrimination was quantified with the area under the receiver operator characteristic (ROC) curve. Calibration was estimated by graphic representation of the associations between observed outcome frequencies and predicted probabilities (calibration curves) for the patient groups. A logistic regression model for positive LNs was developed based on predictor variables: T staging, tumor grade, vascular invasion, and p53 expression. Statistical analyses were performed using two-sided $\mathrm{p}<0.05$ 
as significant. Models, statistics, and figures were prepared using IBM SPSS software version 23.0 (IBM Corp., Armonk, NY, USA) and R 3.2.21 (http://www.cran.r-project.org).

We considered three different external validation methods for the nomogram by Zhu et al. (15): $\mathrm{k}$-fold, leave-one-out, and bootstrap. We sought to validate and verify whether this nomogram was useful for the prediction of positive ILN with good estimates in terms of confidence intervals.

\section{RESULTS}

This study analyzed 65 men with stage cNo (intermediate/high risk) penile cancer who underwent ILND for nonpalpable ILN (Table-1) from 3 institutions in Brazil. The mean age was $56.8 \pm 14.7$ years (range, 25-86 years). Twenty-four (36.9\%) patients presented with positive LNs (Table-1) on ILND. Either standard or modified ILND was performed in all patients. Superficial and deep ILN were removed. A median of 21 (range, 3-60) ILN was removed and the mean number of positive ILN was 2.4 (range, 1-12). T1 stage was observed in $16(24.6 \%)$ patients, T2a in $25(38.5 \%)$, T2b in $7(10.8 \%)$, T3 in $16(24.6 \%)$, and T4 in $1(1.5 \%)$ patient. Low-grade tumor (G1) was observed in 20 $(30.8 \%)$ patients and G2 (61.5\%) in 40 patients (Table-1). Comparing stage and ILN metastases, 8/16 (50\%) patients with T1 stage, 13/32 (40.6\%) with T2 stage, 3/16 (18.8\%) with T3 stage, and 0/1 (0\%) with T4 stage had ILN metastasis, respectively.

Table 1 - Clinicopathological characteristics in 65 patientes with penile cancer.

\begin{tabular}{|c|c|c|c|}
\hline \multirow{3}{*}{$\begin{array}{l}\text { Variables } \\
\text { Age (anos) } \\
\text { Variables }\end{array}$} & & \multicolumn{2}{|c|}{ Mean $\pm d p(\min -m a x)$} \\
\hline & & \multicolumn{2}{|c|}{$56.58 \pm 14.7(25-86)$} \\
\hline & & $\mathrm{N}$ & $(\%)$ \\
\hline \multirow{5}{*}{ T stage } & $\mathrm{T} 1$ & 16 & $(24.6)$ \\
\hline & $\mathrm{T} 2 \mathrm{a}$ & 25 & $(38.5)$ \\
\hline & $\mathrm{T} 2 \mathrm{~b}$ & 7 & $(10.8)$ \\
\hline & T3 & 16 & (24.6) \\
\hline & $\mathrm{T} 4$ & 1 & $(1.5)$ \\
\hline \multirow{3}{*}{ Grade } & G1 & 20 & $(30.8)$ \\
\hline & G2 & 40 & (61.5) \\
\hline & G3 & 5 & $(7.7)$ \\
\hline \multirow{2}{*}{ Lymphovascular invasion } & Absente & 56 & $(86.2)$ \\
\hline & Present & 9 & $(13.8)$ \\
\hline \multirow{2}{*}{ p53 Expression } & Weak & 32 & $(49.2)$ \\
\hline & strong & 33 & $(50.8)$ \\
\hline \multirow{3}{*}{ EAU risk classification } & low & 0 & $(0)$ \\
\hline & Intermediate & 14 & $(21.5)$ \\
\hline & High & 51 & $(78.5)$ \\
\hline \multirow{4}{*}{ pN stage } & NO & 41 & $(63.1)$ \\
\hline & N1 & 11 & $(16.9)$ \\
\hline & N2 & 11 & (16.9) \\
\hline & N3 & 2 & $(3.1)$ \\
\hline \multirow{2}{*}{ Pathologic lymph node status } & positive & 24 & $(36.9)$ \\
\hline & negative & 41 & (63.1) \\
\hline
\end{tabular}


In our study, tumor grade was not associated with LN involvement ( $\mathrm{p}=0.538)$. Regarding histology, we found 30.8\%, 61.5\%, and 7.7\% of tumors to be G1, G2, and G3, respectively (Table-1). On the other hand, only $4.8 \%$ of patients with negative LNs had G3 disease. Lymphovascular invasion was present in $20 \%$ of patients with positive LNs and in 10\% of patients with negative LNs. In univariate analysis tumor grade and lymphovascular invasion were strongly correlated with LN status $(\mathrm{p}<0.05)$. In the multivariate analysis, only $\mathrm{T}$ stage was statistically significant ( $p=0.015$; Table-2).

Our study included the k-fold, leave-one-out, and bootstrap methods to evaluate the nomogram by Zhu et al. (15). The bootstrap method determined that this nomogram is random and does not establish a pattern of prediction of metastasis. Validation using the $\mathrm{k}$-fold method confirmed this, which we identified during the process of modeling. The predictors shown in the nomogram of Zhu et al. (15) were not statistically significant predictors of ILN metastases in our study sample.
All three models showed a low $\mathrm{R}^{2}$ (Table-3). These findings demonstrate that the nomogram by Zhu et al. (15) has a high probability of false negatives in our population. The distribution of the bootstrap test results is shown in Figure-1.

\section{DISCUSSION}

There are some nomograms in literature to predict inguinal lymph nodes, for example, one of them was reported by Ficarra et al. (20) and included variables as tumor thickness, grown pattern, grade, LVI, local infiltration, cN stage. Other one was published recently by Peak (21) that used only grade, cN stage, and LVI. Zhu's nomogram used cT stage, grade, LVI and p53 expression and must be applied in No patients. We decided to validate Zhu's nomogram because of that idea of use a biomarker as p53 expression in association with clinical data, however in our study, this nomogram applied in our population had low accuracy for identifying patients with penile cancer who had positive ILN. Our analysis showed an unde-

Table 2 - Univariate and multivariate analysis of clinicopathological factors to predict inguinal lymph node metastasis in 65 patients.

\begin{tabular}{|c|c|c|c|c|}
\hline \multirow{2}{*}{ Variables } & \multicolumn{2}{|c|}{ Univariate analysis } & \multicolumn{2}{|c|}{ Multivatiate analysis } \\
\hline & $\%$ LNM & $\mathrm{p}$-value & OR $(95 \% \mathrm{Cl})$ & $p$-Value \\
\hline T stage & & 0.154 & & 0.015 \\
\hline $\mathrm{T} 1$ & 50.0 & & & \\
\hline T2a & 32.0 & & $0.341(0.111-1.049)$ & 0.061 \\
\hline $\mathrm{T} 2 \mathrm{~b}$ & 71.4 & & $2.20(0.399-12.120)$ & 0.365 \\
\hline $\mathrm{T} 3$ & 17.6 & & $0.075(0.012-0.462)$ & 0.005 \\
\hline Grade & & 0.010 & & 0.737 \\
\hline $\mathrm{G} 1$ & 35.0 & & & \\
\hline G 2 & 35.0 & & $0.731(0.282-1.893)$ & 0.518 \\
\hline G 3 & 60.0 & & $1.489(0.145-15.235)$ & 0.737 \\
\hline Lymphovascular invasion & & 0.002 & & 0.071 \\
\hline Absente & 33.9 & & & - \\
\hline Present & 55.6 & & $5.965(0.857-41.507)$ & \\
\hline p53 Expression & & 0.350 & & 0.296 \\
\hline Weak & 31.3 & & & - \\
\hline Strong & 42.4 & & $1.789(0.602-5.318)$ & \\
\hline
\end{tabular}


Table 3 - Comparison of results in 3 diferents external validation methods.

\begin{tabular}{lcccc}
\hline Calibration & Zhu's Nomogram & K-fold & Leave-one-out & Bootstrap \\
\hline $\mathrm{R}^{2}$ & 0.445 & $0.228-0.424$ & $0.254-0.389$ & $0.012-0.520$ \\
Brier & 0.116 & $0.170-0.195$ & $0.169-0.186$ & $0.141-0.230$ \\
Discrimination & & & & \\
(ROC) Area under de curve & 0.851 & $*$ & $*$ & 0.783 \\
\hline
\end{tabular}

${ }^{*}$ ROC curve was performed for bootstrap only.

Figure 1 - ROC curve generated by Bootstrap method.

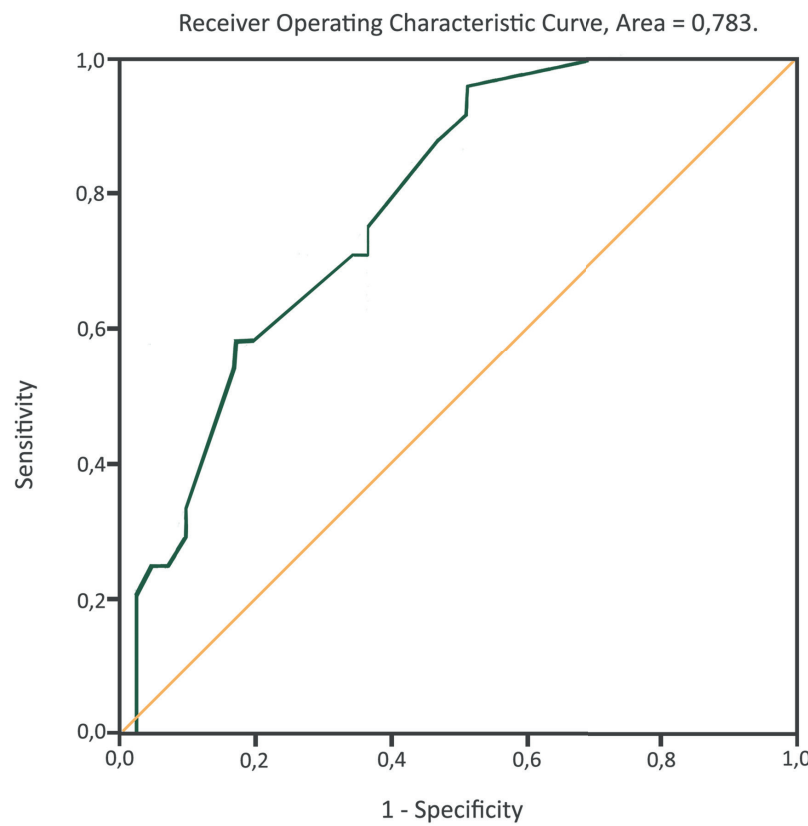

restimation of positive LNs. We would like to emphasize that in using the nomogram by Zhu et al. (15) here, we could not improve the selection of patients with positive or negative ILN.

The occurrence and extent of ILN metastasis are the most important prognostic factors in patients with penile cancer and usually imply worse oncologic prognosis (22). Up to $25 \%$ of patients with no palpable LNs have occult micrometastases that are not detected by physical examination (2325), and imaging studies, such as computed tomography scan or conventional magnetic resonance imaging, are also unable to detect inguinal micrometastases (26). Consequently, it could be debated that lymphadenectomy should be performed for all patients with penile cancer $(8,27)$ because ILN status is the key prognostic factor for survival, and patients can be cured by undergoing ILND. However, this poses a dilemma because early ILND leads to high rates (up to 50\%) (28) of complications with significant morbidity, such as infection and/ or wound dehiscence, skin necrosis, lymphedema, lymphoceles, and other complications. Surveillance strategies can reduce cancer-specific survival (5, 7-9). Patient survival is over 90\% with early lymphadenectomy and less than 40\% in patients treated with a surveillance strategy and later lymphadenectomy for regional recurrence. The alternatives, including DSN $(9,10)$ or minimally invasive approaches such as pure laparoscopic or robotic-assisted ILND (11, 12, 29, 30), are dependent on technology and have high costs, which make them extremely difficult to use in underprivileged populations. Nomograms could be a very interesting tools for improving patient outcome, however in daily practice they are underutilized because the guidelines recommendation of ILND for intermediate- and high-risk tumors (16), other alternatives as DSN and also because of lack of external validation of the available monograms.

Our univariate analysis found that tumor grade and lymphovascular invasion had a strong correlation with LN status. In the multivariate analysis, only $\mathrm{T}$ stage was statistically significant. Lymphovascular invasion was the only statistically significant variable in the study published by Zhu et al. (15) whereas we did not find statistical significance for this variable in our study $(\mathrm{p}=0.212)$. In patients with positive LNs, $20.8 \%$ had lymphovascular invasion; this pathological finding was present in 9.7\% of patients with negative LNs. The 
lymphovascular invasion is a strong predictor of positive inguinal lymph nodes as showed in other studies by Ficarra et al. (31), and other nomogram developed using the National Cancer Database that included 1,636 men in their analysis (21). Our hypothesis is that we found significance only in univariate because of the limited sample.

Zhu et al. (15) developed their nomogram because of the unreliability of currently available modalities for detecting occult nodal involvement, the need for decisive management of regional LNs for improvement of long-term patient survival, and the challenge of avoiding overtreatment with potential treatment-related morbidity. We sought to validate this nomogram for the prediction and identification of patients at risk for nodal metastasis who could potentially be spared unnecessary ILND. In this nomogram, surveillance is recommended if the nomogram probability of positive nodes is $0.1(10 \%)$. The nomogram represents an attempt to define an objective, systematic, standardized, multivariate model capable of providing individual $\mathrm{pN}$ stage predictions. In our study, we performed ILND for cases with intermediate and high risk, according to EAU guidelines. Using this classification, we performed 41 unnecessary ILND and detected 24 cases of ILN metastasis. Considering the threshold of 10\% prediction risk (Zhu et al.) in our study, we had 35 patients (62\%) that underwent ILND unnecessarily (true negatives), and we would have missed $3(12 \%)$ patients with LN metastasis (true positives). Using a threshold of 20\%, 31 (59\%) underwent ILND unnecessarily (true negatives) and we would have missed the same $3(12 \%)$ patients with LN metastases (true positives).

Despite the fact that this nomogram is a noninvasive and low-cost approach, it requires external validation. The aim of the present study was to externally validate a predictive model for ILN metastasis in our cohort of patients who had undergone ILND. Only pN status performed adequately within our external cohort of patients, and this finding was consistent using different statistical means (i.e., overall performance, discrimination, calibration, and clinical usefulness).

The nomogram proposed by Zhu et al. (15) is basically a model that can be used to explain the variability of one or more variables and the association and correlation of this variability with other ex- ploratory variables. The goal is to determine values for the parameters in the specified template that generate the best fit of the model to the data. The best model is the one that produces the least unexplained variability, subject to the restriction that all model parameters must be statistically significant. One of the most important principles concerning the process of modeling is simplification of the model. The principle of parsimony says that given a set of equally good possible explanations, the correct explanation is the simplest one. Accordingly, given a set of valid models, the best model is the one that: a) includes the least number of variables, B) is linear and contrasts with nonlinear models, C) is based on few statements, and D) recognizes that simple explanations are always preferable in comparison with complex explanations. In the case of the model proposed by Zhu et al. (15), only lymphovascular invasion was identified as a statistically significant predictor for positive ILN. We used the bootstrap method because this method is used to estimate the confidence interval of parameters. In the bootstrap method, we set the answer and performed resampling of predictors (1,000 times) to identify confidence intervals for the parameters of the logistic regression and to identify better and greater values for $\mathrm{R}^{2}$, the c-index statistic, and Brier score. Using the k-fold validation method, we measured the accuracy of the model, i.e., the model's ability to faithfully represent the sample data. We used a third-party validation method, the leave-one-out method, which is a generalization of the k-fold method, where the number of templates is equal to the size of the sample. The method is useful for evaluating the complete behavior of the model and for correcting defects of the model. Considering that, we identified the extremes of confidence intervals for the parameters of logistic regression. Again, we identified the values of $\mathrm{R}^{2}$ statistics, the c-index, and Brier score. These analyses confirmed that in our sample, the model proposed by Zhu et al. (15) was inappropriate, and even cross-validation did not improve the model. In our sample, the predictors shown in the nomogram of Zhu et al. (15) were not statistically significant predictors of ILN. All models showed a low $\mathrm{R}^{2}$, including with the bootstrap technique (between 0.228 and 0.424) and leave-one-out (between 0.254 and 0.389) method. In the bootstrap method, p53 expression was identified as a better parameter. 
We found that accuracy of this nomogram was lower in our sample (area under the ROC curve, 0.79). The calibration plot showed underestimation of positive ILN. This indicates poor sensitivity, poor specificity, and a low positive likelihood ratio for the various values used in the nomogram by Zhu et al. (15). According to our findings, we would like to highlight that the nomogram by those authors does not have satisfactory performance in improving selection of patients with positive or negative ILN disease, even using a threshold of $10 \%$ or $20 \%$. The applicability of models derived from cohorts in China may be questionable when transferred to Latin America. These results could be explained for some reasons: different population and race, low accuracy of Zhu's nomogram, limited sample, lack of other biomarkers, etc.

The limitations of the present study are inherent to any retrospective series. The number of patients was small $(\mathrm{N}=65)$; however, considering the rarity of penile cancer, our sample size is similar to those in other published series in the literature. Our population was significant and sufficient for validation of the nomogram by $\mathrm{Zhu}$ et al. $(\mathrm{N}=110)$ in penile cancer. Lymphadenectomy templates were not standardized; however, the three institutions and the surgeons involved are experts in urologic oncology and have extensive experience in the management of penile cancer. Nevertheless, our data reflect a real-world, multicenter experience.

\section{CONCLUSIONS}

In our study, the nomogram by Zhu et al. (15) applied in our population had low accuracy and low precision for correctly identifying patients with penile cancer who have positive ILN. Our analysis showed an underestimation of positive LNs. Using this nomogram, we could not improve the selection of patients with positive versus negative ILN.

\section{ABBREVIATIONS}

$\mathrm{PC}=$ Penile cancer

$\mathrm{LN}=$ Lymph node

ILN = Inguinal LN (ILN)

DSNB = dynamic sentinel node biopsy

ILND = Inguinal LN dissection
EAURC $=$ European Association of Urology Risk Classification

\section{ACKNOWLEDGEMENTS}

Rafael José de Souza, MD; Renato Abreu Faria, MD; José Humberto Fregnani, MD

\section{CONFLICT OF INTEREST}

None declared.

\section{REFERENCES}

1. Backes DM, Kurman RJ, Pimenta JM, Smith JS. Systematic review of human papillomavirus prevalence in invasive penile cancer. Cancer Causes Control. 2009;20:449-57.

2. Chaux A, Netto GJ, Rodríguez IM, Barreto JE, Oertell J, Ocampos S, et al. Epidemiologic profile, sexual history, pathologic features, and human papillomavirus status of 103 patients with penile carcinoma. World J Urol. 2013;31:8617.

3. Pandey D, Mahajan V, Kannan RR. Prognostic factors in node-positive carcinoma of the penis. J Surg Oncol. 2006;93:133-8.

4. Srinivas SV, Lynch DF Jr. Summary of penile cancer: relation of extent of nodal metastasis to survival (by Sri V. Srinivas, MD, M.J. Morse, MD, H.W. Herr, MD, P.C. Sogani, MD, and W.F. Whitmore, Jr, MD). 1987. Semin Urol Oncol. 1997;15:136-9.

5. Ornellas AA, Seixas AL, de Moraes JR. Analyses of 200 lymphadenectomies in patients with penile carcinoma. J Urol. 1991;146:330-2.

6. Ravi R. Correlation between the extent of nodal involvement and survival following groin dissection for carcinoma of the penis. Br J Urol. 1993;7(5Pt 2):817-9.

7. Kroon BK, Horenblas S, Lont AP, Tanis PJ, Gallee MP, Nieweg $\mathrm{OE}$. Patients with penile carcinoma benefit from immediate resection of clinically occult lymph node metastases. J Urol. 2005;173:816-9.

8. Ornellas AA, Seixas AL, Marota A, Wisnescky A, Campos F, de Moraes JR. Surgical treatment of invasive squamous cell carcinoma of the penis: retrospective analysis of 350 cases. J Urol. 1994;151:1244-9.

9. Kroon BK, Horenblas S, Deurloo EE, Nieweg OE, Teertstra HJ. Ultrasonography-guided fine-needle aspiration cytology before sentinel node biopsy in patients with penile carcinoma. BJU Int. 2005;95:517-21. 
10. Perdonà S, Autorino R, De Sio M, Di Lorenzo G, Gallo L, Damiano $\mathrm{R}$, et al. Dynamic sentinel node biopsy in clinically node-negative penile cancer versus radical inguinal lymphadenectomy: a comparative study. Urology. 2005;66:1282-6.

11. Tauber R, Schmid S, Horn T, Thalgott M, Heck M, Haller B, et al. Inguinal lymph node dissection: epidermal vacuum therapy for prevention of wound complications. J Plast Reconstr Aesthet Surg. 2013;66:390-6.

12. Matin SF, Cormier JN, Ward JF, Pisters LL, Wood CG, Dinney CP, et al. Phase 1 prospective evaluation of the oncological adequacy of robotic assisted video-endoscopic inguinal lymphadenectomy in patients with penile carcinoma. BJU Int. 2013;111:1068-74.

13. Pahwa HS, Misra S, Kumar A, Kumar V, Agarwal A, Srivastava R. Video Endoscopic Inguinal Lymphadenectomy (VEIL)--a prospective critical perioperative assessment of feasibility and morbidity with points of technique in penile carcinoma. World $\mathrm{J}$ Surg Oncol. 2013;11:42.

14. Zhou XL, Zhang JF, Zhang JF, Zhou SJ, Yuan XQ. Endoscopic inguinal lymphadenectomy for penile carcinoma and genital malignancy: a preliminary report. J Endourol. 2013;27:657-61.

15. Zhu Y, Zhang HL, Yao XD, Zhang SL, Dai B, Shen YJ, et al. Development and evaluation of a nomogram to predict inguinal lymph node metastasis in patients with penile cancer and clinically negative lymph nodes. J Urol. 2010;184:539-45.

16. Hakenberg OW, Compérat EM, Minhas S, Necchi A, Protzel C, Watkin N. EAU guidelines on penile cancer: 2014 update. Eur Urol. 2015;67:142-150.

17. Penis. In: AJCC Cancer Staging Manual, 6th ed.American Joint Committee on Cancer. New York: Springer 2002; pp. 303-8.

18. Broders AC. Squamous-cell epithelioma of the skin: a study of 256 cases. Ann Surg. 1921;73:141-60.

19. Zhu Y,Zhou XY, Yao XD, Dai B, Ye DW. The prognostic significance of p53, Ki-67, epithelial cadherin and matrix metalloproteinase-9 in penile squamous cell carcinoma treated with surgery. BJU Int. 2007;100:204-8.

20. Ficarra V, Zattoni F, Artibani W, Fandella A, Martignoni G, Novara $\mathrm{G}$, et al. Nomogram predictive of pathological inguinal lymph node involvement in patients with squamous cell carcinoma of the penis. J Urol. 2006;175:1700-4; discussion 1704-5.

21. Peak TC, Russell GB, Dutta R, Rothberg MB, Chapple AG, Hemal AK. A National Cancer Database-based nomogram to predict lymph node metastasis in penile cancer. BJU Int. 2018; 11. [Epub ahead of print]

22. Abi-Aad AS, deKernion JB. Controversies in ilioinguinal lymphadenectomy for cancer of the penis. Urol Clin North Am. 1992;19:319-24.
23. Lont AP, Kroon BK, Gallee MP, van Tinteren H, Moonen LM, Horenblas S. Pelvic lymph node dissection for penile carcinoma: extent of inguinal lymph node involvement as an indicator for pelvic lymph node involvement and survival. $J$ Urol. 2007;177:947-52; discussion 952.

24. Slaton JW, Morgenstern N, Levy DA, Santos MW Jr, Tamboli P, Ro JY, et al. Tumor stage, vascular invasion and the percentage of poorly differentiated cancer: independent prognosticators for inguinal lymph node metastasis in penile squamous cancer. J Urol. 2001;165:1138-42.

25. Protzel C, Alcaraz A, Horenblas S, Pizzocaro G, Zlotta A, Hakenberg OW. Lymphadenectomy in the surgical management of penile cancer. Eur Urol. 2009;55:1075-88.

26. Singh AK, Gonzalez-Torrez P, Kaewlai R, Tabatabaei S, Harisinghani MG. Imaging of penile neoplasm. Semin Ultrasound CT MR. 2007;28:287-96.

27. Spiess $P E$, Hernandez MS, Pettaway CA. Contemporary inguinal lymph node dissection: minimizing complications. World J Urol. 2009;27:205-12.

28. Stuiver MM, Djajadiningrat RS, Graafland NM, Vincent $A D$, Lucas C, Horenblas S. Early wound complications after inguinal lymphadenectomy in penile cancer: a historical cohort study and risk-factor analysis. Eur Urol. 2013;64:486-92.

29. Tobias-Machado M. Video endoscopic inguinal lymphadenectomy (VEIL): is a new standard ready to be accepted? BJU Int. 2017;119:504-5.

30. Tobias-Machado M, Tavares A, Molina WR Jr, Forseto PH $\mathrm{Jr}$, Juliano RV, Wroclawski ER. Video endoscopic inguinal lymphadenectomy (VEIL): minimally invasive resection of inguinal lymph nodes. Int Braz J Urol. 2006;32:316-21.

31. Ficarra V, Zattoni F, Cunico SC, Galetti TP, Luciani L, Fandella A, et al. Lymphatic and vascular embolizations are independent predictive variables of inguinal lymph node involvement in patients with squamous cell carcinoma of the penis: Gruppo Uro-Oncologico del Nord Est (Northeast Uro-Oncological Group) Penile Cancer data base data. Cancer. 2005;103:2507-16.

Correspondence address:

Eliney Ferreira Faria, MD

Departamento de Urologia, Hospital do Câncer de Barretos

Rua Antenor Duarte Vilela, 1331 - Bairro Dr Paulo Prata Barretos, SP, 14784.400, Brasil Telephone: +55 17 3321-6600 E-mail: elineyferreirafaria@yahoo.com.br 